\title{
Memory effects of motor activation in subject-performed tasks and sign language
}

\author{
JAN D. vON ESSEN and LARS-GÖRAN NILSSON \\ Stockholm University, Stockholm, Sweden
}

\begin{abstract}
Research on memory has consistently shown that when a subject-performed task (SPT) is compared with a traditional verbal task (VT), enactment at the encoding of verbal materials (i.e., SPT) yields better memory performance than does nonenactment (i.e. VT). There is some controversy regarding the extent to which motor activation per se might be causing this effect, and whether or not SPTs may be influenced by memory strategies. The purpose of this study was to contribute toward a solution of these questions. The effect of SPT encoding was compared with the effect of encoding by means of a sign language task (SLT). The SLT condition is claimed to be a verbal/linguistic task with a major relevant motor component. The motor activation in SLT is in the present study seen to be the main difference between the SLT and the VT, and the main similarity between the SLT and the SPT. Control conditions were testedin order to evaluate possible effects of translation and imagery in the SLT condition. Subjects in the SLT condition performed similarly to subjects in the SPT condition in free recall. Subjects in both these conditions outperformed subjects in the control conditions. The SPT and SLT superiority is suggested to be caused mainly by relevant motor activation.
\end{abstract}

Research on memory has consistently shown that enactment of verbal materials at the time of encoding enhances memory performance relative to that obtained in a traditional verbal task (VT). This paradigm is referred to as an action memory task or a subject-performed task (SPT). In SPT encoding, subjects are required to enact the verbal to-be-remembered (TBR) items, which consist of instructions or action phrases, by performing minitasks (e.g., split the apple, water the plant). This manipulation results in superior recall and recognition in SPTs as opposed to VTs. (See Cohen, 1989; Nilsson, 2000, for reviews.) After over 20 years of research, the causes for the phenomenon are still not quite understood.

Three different theories were proposed early by different groups of scientists to account for this "SPT effect" (Bäckman \& Nilsson, 1984; Cohen, 1981; Engelkamp \& Zimmer, 1984, 1985). More recently, these authors have expressed similar theoretical positions, in which physical movement is seen as a crucial component of SPT encoding. They also consider the encoding of SPTs to be relatively independent of memory strategies (i.e., it is nonstrategic; Nilsson, 2000).

The notion of a nonstrategic nature for SPT encoding arose from studies in which the classical benefits of memory strategies for verbal encoding could not be shown for items encoded by enactment. Recall of SPTs has been

This research was supported by a grant from the Swedish council of research for the humanities and social sciences. Correspondence concerning this article should be addressed to J. von Essen, Department of Psychology, Stockholm University, SE-10691 Stockholm, Sweden (e-mail: jve@psychology.su.se). shown to lack a primacy effect (Bäckman \& Nilsson, 1984; Cohen, 1981), to be uninfluenced by rate of presentation (Cohen, 1985), and not to be helped by subjects' generating the TBR items on their own (Nilsson \& Cohen, 1988).

The importance of physical movement per se for the SPT effect was first stressed by Engelkamp and Zimmer (1984, 1985). They proposed a third "program" that governed SPT encoding. The proposed motor program was said to be independent of the earlier proposed verbal and visual programs, with different representations, codes, and modality-specific properties. They also claimed that motor encoding is more efficient than verbal and visual encoding, thereby causing the enactment effect. The actual mechanism for this higher efficiency of motor encoding, however, was not specified by Engelkamp and Zimmer (1984, 1985). Support for their claims has, for example, been shown in studies where the rate of false alarms at recognition was increased by motorically similar distractors (Engelkamp \& Zimmer, 1994, 1995). This result would be expected if subjects reprocess motor information during retrieval, and if the reprocessing has been influenced by motorically similar distractors. Studies in which the SPT effect has been found without sensory input of seeing and/or touching at encoding (e.g., Engelkamp, Zimmer, \& Biegelmann, 1993) also support these claims, since this rules out the effect of multimodal encoding of visual and tactile information.

Contrary to the theoretical positions mentioned above, Kormi-Nouri (1995) presented a theory in which the motor component is not crucial for the SPT effect. According to Kormi-Nouri, the SPT effect is based on higher selfinvolvement, self-actualization, or self-reference of subjects during learning. These factors cause an increased 
integration within and between action events at encoding, which Kormi-Nouri named episodic integration. Within the events, the integration is increased between the verb and the noun, so that these are encoded together as one memory unit (or as two closely connected units).

Kormi-Nouri (1995) based this conclusion that physical movement is of little importance on studies showing that SPT encoding could be influenced by verbal or visual distractors (Bäckman, Nilsson, \& Kormi-Nouri, 1993; Kormi-Nouri, Nilsson, \& Bäckman, 1994). Also, KormiNouri, Nyberg, and Nilsson (1994) found that performing the TBR action at recognition did not enhance the SPT effect, which had been the case for Engelkamp, Zimmer, Mohr, and Sellen (1994).

The empirical inconsistencies regarding the role of actual movement in accounting for the enactment effect call for additional clarification of this issue. One way to study the effect of movement per se is to study movement comparable to movement in SPT, without the performing of an action. Schaaf (1988) demonstrated that performing an irrelevant action did not result in an SPT effect. This result suggests that movement has to be relevant in some way to be comparable to movement in SPT. Sign languages are in this sense an interesting area, since they consist of extensive motor activity which is relatively easy to control and highly relevant-not for performing an action but for producing a verbal sentence.

Sign languages have been recognized as verbal communication for some time (Emmorey \& Lane, 2000; Klima \& Bellugi, 1979). Several authors have argued that American Sign Language is as complex and systematic a language as any spoken one (Emmorey \& Lane, 2000; Messing \& Campbell, 1999). Similar research has been conducted on other sign languages, all of which are unanimously considered real languages. Today, sign languages are considered to be qualitatively similar to spoken language rather than to nonverbal communication through gestures (Emmorey \& Lane, 2000; Messing \& Campbell, 1999). This motivates the use of sign languages just as any other language in verbal memory tests.

Research directly concerned with effects of sign language production on hearing subjects' long-term memory is rare. To the authors' knowledge, classical verbal episodic memory tests, or SPT studies, have not yet been compared with tests in which subjects use sign language. Presently, however, there is another research group exploring similarities between SPT and sign language (Zimmer \& Engelkamp, 2001). The preliminary results of their study indicate that signing at encoding (SLT) enhances memory as well as does performing actions at encoding (SPT).

In addition to any possible effects of motor activation, two aspects of the SLT condition, in particular, require attention. One comprises the potential effects of translation. When asked to sign a sentence, subjects must perform a translation from their first language into their second (or vice versa). It has been suggested that mem- ory effects of translation resemble generation effects, consisting of enhanced memory performance due to conceptual processing (see Francis, 1999, for a review). But the results are contradictory. In studies with intentional learning instructions, no translation effect has been observed (see Francis, 1999). One control condition in which Swedish had to be translated into English was therefore included in the present study, so that we could examine translation effects (translation task, TT).

The other interfering aspect is the potential role of motor imagery. When asked to sign the sentence roll the pen, for example, subjects are forced to interpret the action of the sentence, because one would sign it differently if one rolled the pen on a table or between one's fingers. Such an interpretation can take the form of visual or of motor imagery. Some sentences (items) are less likely to demand such interpretation, as, for example, sentences in which a single way of performing the action is more common than others (e.g., read the book). Imagery has long been recognized as an effective mnemonic strategy (Paivio, 1979). The effect of motor imagery on memory of events has been studied in parallel with the SPT effect by Denis, Engelkamp, and Mohr (1991) and by Nilsson et al. (2000). The conclusion suggested by these papers is that motor imagery affects memory of actions in a similar way as does SPT, but to a lesser degree. Therefore, the subjects in two of the control conditions in the present study (imagery task, IT, and translation task, TT) were asked to imagine themselves performing the action denoted in the commands, so that we could evaluate the effect of motor imagery in signing. If the SLT condition outperformed the control conditions (IT and TT), the advantage could not be due to motor imagery in the SLT condition.

The purpose of the present study was to explore whether the enacted encoding in SPT and the linguistic encoding (including movement) in SLT would have different effects on recall performance. Since the SLT group was performing a verbal task, we hypothesized that similarities in memory performance between the SLT group and the SPT group would be attributable to performing relevant movement.

\section{METHOD}

\section{Design}

The design of the study was 5 (type of encoding: subject performed task, verbal task, sign language task, translation task, and imagery task) $\times 2$ (type of integration: well integrated or poorly integrated). The first factor was varied between subjects. Originally, a within-subjects manipulation of semantic integration, was included in the design. However, this manipulation showed little effect and is therefore not included in the analyses.

\section{Subjects}

A total of 75 students, 58 women and 17 men 18-47 years old, participated in the study. The sign language task (SLT) group consisted of 11 hearing women from the senior class of the Swedish sign language (SSL) education at Bredviken Gymnasium. To be accepted for this education, the applicants had to have some command 
of SSL. The education consists of 2 years of improving their knowledge of SSL grammar and practice. Thus, the SLT subjects had Swedish as primary language and SSL as secondary language. Because of practical limitations in contacting hearing subjects with sufficient sign language skills, all the 25 students in the senior class were gathered for a brief presentation. They were introduced to issues about scientific ethics and confidentiality, and offered SEK 50 for their participation. The task was explained as consisting of signing short, easy sentences and trying to recall them at testing. This explanation was given without revealing the purpose or design of the study.

The other subjects were psychology students at Stockholm University. They all volunteered to participate in a study of memory. Sixty-four subjects were randomly assigned to the four remaining conditions (SPT, IT, TT, and VT). The TT subjects were expected to have command of English comparable to the SLT subjects' command of signing, which is normal at Swedish universities. Their actual level of knowledge was, however, not tested. Their performance at translating the items in the experiment was as expected. One subject from the SLT condition and 1 from the IT condition were deleted because they were unable to complete the experiment according to instructions. Thus, there were 16 subjects in the SPT, TT, and VT conditions, 15 in IT, and 10 in SLT.

\section{Materials}

The to-be-remembered (TBR) items consisted of 60 sentences. The items were constructed in co-operation with a signing advisor, and were tested for usability by 3 subjects prior to the actual experiment. The order of the items were randomly assigned to three lists, one of which was randomly assigned to each subject. A vocabulary test (see Nilsson et al., 1997) was used as an interpolated task between study and test of the to-be-remembered items, and as a means to assess word comprehension. The interstimulus time was controlled by signals recorded on audiotape, hearable only to the experimenter.

\section{Procedure}

All the subjects were tested one by one. The items were presented verbally by the experimenter, at an interstimulus interval of $10 \mathrm{sec}$. SPT subjects were instructed to perform the action indicated by the items, with imaginary objects. The SLT subjects were instructed to sign the items back to the experimenter, as if communicating spontaneously. The IT subjects were instructed to imagine themselves performing the action. The TT subjects were instructed to first imagine themselves performing the action, and immediately after this, to repeat the sentence in English, as if spontaneously communicating. The reason for adding brief imagery to the translation task was to make the motor activation the main difference between SLT and TT. The VT subjects were only instructed to remember the verbal sentences. The subjects in all conditions were instructed to remember the verbal sentences, for intentional learning conditions. To encourage spontaneous signing by SLT subjects, and relaxed translation by TT subjects, the experimenter ensured that their translating or signing abilities would not be evaluated.

Before the actual study list was presented, the subjects were given two items for practice, with the correct interstimulus interval. When all instructions were understood, the TBR items were presented. After the presentation of the last item, the subjects were given a 30-item vocabulary test, in order to eliminate any effects of short-term memory. The test was also used to assess the subjects' word comprehension. Seven minutes were allowed for completing the vocabulary test. Following the vocabulary test, the subjects were asked to write down as many sentences (items) as they could remember, as correctly as possible. Fifteen minutes were allowed for recalling items. The experimenter immediately made sure that he could read all the sentences that the subjects had written down. Finally the subjects were asked to complete a short list of general feedback questions. The whole procedure took about $40 \mathrm{~min}$.

\section{RESULTS}

The mean probabilities of correct recall, with correct verbs and correct nouns, for the different conditions are presented in Figure 1. The results in Figure 1 show the highest probabilities of recall for the SPT $(M=.54)$ and SLT $(M=.52)$ conditions. Furthermore, the recall probability is higher for the IT $(M=.38)$ condition than for the VT $(M=.31)$ and TT $(M=.31)$ conditions.

An analysis of variance (ANOVA) was performed in order to evaluate the data in Figure 1. The results of the ANOVA showed a significant difference between conditions in recall probability $[F(4,68)=9.38, p<.001]$. Scheffé post hoc analyses revealed a significantly higher $(p<.05)$ performance for the SPT and SLT conditions than for the TT and VT conditions. Also, the SPT condition performed significantly higher $(p<.05)$ than the IT condition, whereas the difference between the SLT and the IT conditions did not reach a significant level $(p>.24)$.

The results also indicate a gender difference. The women's mean recall $(M=.43)$ was close to significantly higher $(p<.051)$ than the mean recall for the men $(M=$ $.33)$. Because the male subjects were unevenly distributed in different encoding conditions (from 0 in the SLT condition to 7 in the VT condition), the recall data were reanalyzed using an analysis of covariance (ANCOVA) with gender as covariate (female $=1$, male $=2$ ). The results of the ANCOVA showed a significant difference between conditions in recall probability, for the corrected model $[F(5,67)=7.67, p<.001]$. The ANCOVA showed an insignificant effect $(p>.34)$ for gender as covariate. The corrected means yielded results virtually identical to those shown in Figure 1.

The only differences found between the conditions on the general feedback questions and control variables oc-

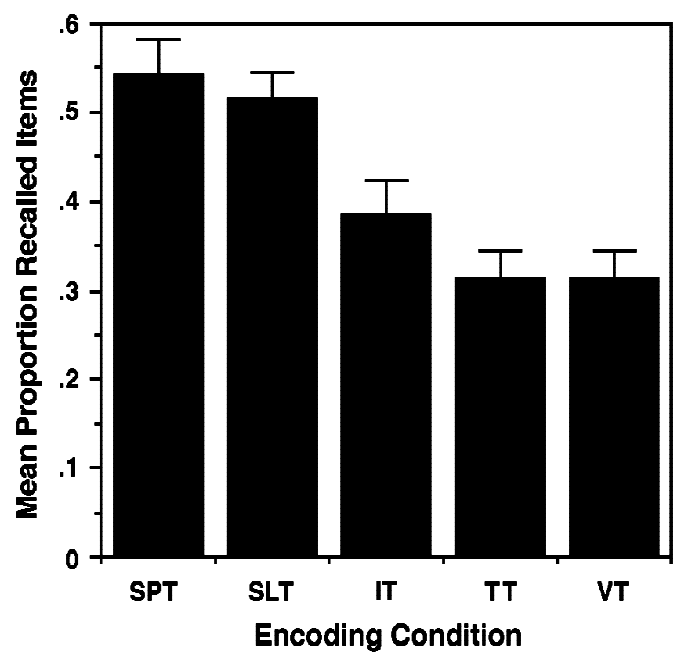

Figure 1. Mean proportions of recalled items for subjects encoding subject-performed tasks (SPT), sign language tasks (SLT), imagery tasks (IT), translation tasks (TT), and traditional verbal tasks (VT). 
curred in self-reported perceived level of difficulty $[F(4,68)=7.88, p<.001]$. Scheffé post hoc analyses showed a significantly higher $(p<.05)$ rating of difficulty for the TT $(M=5.06)$ and VT $(M=4.88)$ conditions over the SLT $(M=2.70)$ conditions.

\section{DISCUSSION}

In accordance with findings in earlier studies, the SPT group outperformed the VT group in recall of TBR items. In addition to this well-documented SPT effect, the results also indicate an SLT effect, in demonstrating that the SLT group also outperformed the VT group. Recall performance in the SPT and the SLT groups did not differ. The similarities in SPT results and SLT results suggest that these two conditions have a similar effect on the memory process. Both conditions served as cognitive support at encoding. This interpretation is also in line with preliminary results found by Zimmer and Engelkamp (2001).

Regarded as experimental conditions, the similarity between SPT and SLT is a relevant motor activation. There is no reason to assume a higher degree of selfinvolvement for the SLT group than for the VT group, which was suggested as the cause of SPT superiority over VT by Kormi-Nouri (1995). Furthermore, the SLT effect is likely not due to the effect of translation, since the TT subjects performed similarly to the VT subjects (i.e., significantly lower than the SLT subjects). The lack of a translation effect is in line with previous studies of translation under intentional learning conditions (see Francis, 1999, for a review). Therefore, it is likely that relevant motor activity may account for the obtained results.

This interpretation contradicts Kormi-Nouri's (1995) theses claiming motor activity to be unimportant for the SPT effect. The results for the SLT group support Engelkamp and Zimmer $(1984,1985)$ in their claim that SPT items are motorically encoded. Given the contradictory results regarding motor encoding, it is not necessary for SPT or SLT encoding to consist of motor memory traces. The main contribution of motor activity can instead consist of cognitive support enhancing the verbal information. This could be in the shape of what Kormi-Nouri calls episodic integration. In the authors' view, these theories do not necessarily contradict each other on this point.

The visualization was included in the IT condition because it is reasonable to assume some cognitive effects, for the SLT group, arising from the subjects' having to create a mental picture of the action, in order to sign the sentence correctly. In order to control for the effect of possible motor imagery in the SLT condition, effort was made to produce a similar cognitive effect in two control groups (IT and TT). The subjects in the IT and TT groups were instructed to imagine themselves performing the indicated action. The subjects in both IT and TT conditions recalled less items than did the SPT subjects and SLT subjects. Although the average performance for the SLT subjects was only slightly lower than that for the SPT subjects, the SPT/IT difference is significant, whereas the SLT/IT difference is not. This is probably due to the lower number of subjects in the SLT condition than in the SPT condition. It seems clear that the SLT result is more similar to the SPT result than to the IT result, indicating an SLT effect regardless of motor imagery.

The result for the IT condition may reflect the importance of motor planning, even though IT performance was not significantly higher than TT and VT performance. It has been suggested that motor imagery (without performing action) and motor preparation are identical (see Decety, 1996, for a review). Nilsson et al. (2000) have recently shown that this kind of imagery does have some positive effect on memory performance in an IT condition. Furthermore, Nilsson et al. (2000) demonstrated that the amount of brain activity, as measured by PET (positron emission tomography), reflects the level of memory performance in SPT and VT, as well as in VT with imagery. The present results are similar to general patterns obtained for the relationship between SPT, VT with imagery (IT), and normal VT (see Nilsson et al., 2000). Hence, the SLT effect is probably not due to motor imagery only, and neither is the SPT effect, because both these conditions outperform the IT condition. Motor planning should, however, be considered an inseparable part of performing SPTs and SLTs.

Subjects in the SLT condition reported experiencing the experiment as more difficult than the TT and VT subjects did. Note that the feedback questions were presented at the end of the experiment, after the subjects had tried to recall the presented items. Therefore, subjects' reports of experienced difficulty may reflect their performance, rather than difficulties experienced during encoding. This hypothesis is supported by a significant negative correlation $(r=-.42, p<.01)$ between items recalled and experienced level of difficulty.

There may have been differences between the subjects in the different conditions prior to the experiment, which should be kept in mind. The signing subjects, after all, could not be sampled from the same population as the SPT and VT subjects. Thus there is the risk that the SLT subjects, with their unique experiences, had a different relation to perceiving and producing motor activity. Deaf subjects were not used in the SLT condition because their experience and perception of producing arm movements (signing) were at the time of planning perceived to further increase the differences between conditions.

The distribution of male and female subjects was uneven between encoding conditions. The SLT group had no male subjects, the SPT group had 3, the IT group had 1 , the TT group had 5, and the VT group had 7. As should be expected in episodic memory tests, the female subjects outperformed the male subjects. Because of the uneven distribution of male subjects, the means were corrected using gender as covariate. The general picture of the corrected recall means supports the earlier conclusion, that the SPT and SLT conditions showed the 
highest performance, the TT and VT showed the lowest performance, and the IT condition performed intermediately.

The extent to which sign language differs from spoken language in both production and representation in memory (relevant for this study) is far from fully understood. The same goes for effects of combining spoken stimuli with reproduction in sign language. These are important issues to keep in mind when one is interpreting these results. It seems, however, that the cognitive processes are much the same for sign language production and for spoken language production (Emmorey \& Lane, 2000; Messing \& Campbell, 1999) —except for the motor component, which is unique to sign language (in this experimental condition). Thus, with visualization of TBR actions in the IT and TT conditions, and translation of TBR items into English in the TT condition, motor activity should be the crucial difference between the SLT and the control conditions. In a similar vein, motor activity and to some degree motor preparation should be considered as the crucial similarities between SPT and SLT conditions, and therefore as causing enhanced memory performance by providing effective cognitive support at encoding.

\section{REFERENCES}

Bäckman, L., \& Nilsson, L.-G. (1984). Ageing effects in free recall: An exception to the rule. Human Learning, 3, 53-69.

Bäckman, L., NiLsson, L.-G., \& Kormi-Nouri, R. (1993). Attentional demands and recall of verbal and color information in action events. Scandinavian Journal of Psychology, 34, 246-254.

Cohen, R. L. (1981). On the generality of some memory laws. Scandinavian Journal of Psychology, 22, 267-281.

Cohen, R. L. (1985). On the generality of the laws of memory. In L.-G. Nilsson \& T. Archer (Eds.), Perspectives on learning and memory (pp. 247-277). Hillsdale, NJ: Erlbaum.

CoHEN, R. L. (1989). Memory for action events: The power of enactment. Educational Psychology Review, 1, 57-80.

Decety, J. (1996). Do imagined and executed actions share the same neural substrate? Cognitive Brain Research, 3, 87-93.

Denis, M., Engelkamp, J., \& Mohr, G. (1991). Memory of imagined actions: Imagining oneself or another person. Psychological Research, 3, 246-250.

EMmorey, K., \& LANe, H. L. (2000). The signs oflanguage revised: An anthologyto honor Ursula Bellugi and Edward Klima. Mahwah, NJ: Erlbaum.

ENGELKAMP, J., \& Zimmer, H. D. (1984). Motor programme information as a separable memory unit. Psychological Research, 46, 283-299.
EngelKamp, J., \& Zimmer, H. D. (1985). Motor programs and their relation to semantic memory. German Journal of Psychology, 9, 239254.

EngelKamp, J., \& Zimmer, H. D. (1994). Motor similarity in subjectperformed tasks. Psychological Research, 57, 47-53.

ENGELKAMP, J., \& Zimmer, H. D. (1995). Similarity of movement in recognition of self-performed tasks and of verbal tasks. British Journal of Psychology, 86, 241-252.

EngelKamp,J., Zimmer, H. D., \& Biegelmann,U. E. (1993). Bizarreness effects in verbal and subject-performed tasks. European Journal of Cognitive Psychology, 5, 393-415.

Engelkamp, J., Zimmer, H. D., Mohr, G., \& Sellen, O. (1994). Memory of self-performed tasks: Self-performing during recognition. Memory \& Cognition, 22, 34-39.

FrancIS, W. S. (1999). Cognitive integration of language and memory in bilinguals: Semantic representation. Psychological Bulletin, 125, 193-222.

Klima, E. S., \& Bellugi, U. (1979). The signs oflanguage. Cambridge, MA: Harvard University Press.

Kormi-Nouri, R. (1995). The nature of memory for action events: An episodic integration view. European Journal of Cognitive Psychology, 7, 337-363.

Kormi-Nouri, R., Nilsson, L.-G., \& Bäckman, L. (1994). The dual conception view re-examined: Attentional demands and the encoding of verbal and physical information in action events. Psychological Research, 51, 181-187.

Kormi-Nouri, R., Nyberg, L., \& Nilsson, L.-G. (1994). The effect of retrieval enactment on recall of subject-performed tasks and verbal tasks. Memory \& Cognition, 22, 723-728.

Messing, L. S., \& Campbell, R. (1999). Gesture, speech, and sign. Oxford: Oxford University Press.

NiLsson, L.-G. (2000). Remembering actions and words. In F. I. M. Craik \& E. Tulving (Eds.), The Oxford handbookof memory (pp. 137148). Oxford: Oxford University Press.

Nilsson, L.-G., Bäckman, L., Erngrund, K., Nyberg, L., Adolfsson, R., Bucht, G., Karlsson, S., Widing, M., \& Winblad, B. (1997). The Betula prospective cohort study: Memory, health, and aging. Aging, Neuropsychology, \& Cognition, 4, 1-32.

NiLsson, L.-G., \& Cohen, R. L. (1988). Enrichment and generation in the recall of enacted and non-enacted instructions. In M. M. Gruneberg, P. E. Morris, \& R. N. Sykes (Eds.), Practical aspects of memory: Current research and issues (Vol. 1, pp. 427-432). Chichester, U.K.: Wiley.

Nilsson, L.-G., Ny berg, L., Klingberg, T., Ảberg, C., Persson, J., \& Roland, P. E. (2000). Activity in motor areas while remembering action events. NeuroReport, 11, 2199-2201.

PaIvio, A. (1979). Imagery and verbal processes. Hillsdale, NJ: Erlbaum. SCHAAF, M. C. (1988). Motor activity and verbal learning: Does simultaneity improve performance? Zeitschrift für Experimentelle und Angewandte Psychologie, 35, 298-302.

Zimmer, H. D., \& ENGelKamp, J. (2001). Signing enhances memory like performing actions. Manuscript in preparation.

(Manuscript received January 17, 2001; revision accepted for publication April 12, 2002.) 